PROCEEDINGS OF THE

AMERICAN MATHEMATICAL SOCIETY

Volume 130, Number 1, Pages 267-270

S 0002-9939(01)06372-9

Article electronically published on July 25, 2001

\title{
ON THE COHOMOLOGY OF GENERALIZED HOMOGENEOUS SPACES
}

\author{
J. P. MAY AND F. NEUMANN \\ (Communicated by Ralph Cohen)
}

\begin{abstract}
We observe that work of Gugenheim and May on the cohomology of classical homogeneous spaces $G / H$ of Lie groups applies verbatim to the calculation of the cohomology of generalized homogeneous spaces $G / H$, where $G$ is a finite loop space or a $p$-compact group and $H$ is a "subgroup" in the homotopical sense.
\end{abstract}

We are interested in the cohomology $H^{*}(G / H ; R)$ of a generalized homogeneous space $G / H$ with coefficients in a commutative Noetherian $\operatorname{ring} R$. Here $G$ is a "finite loop space" and $H$ is a "subgroup". More precisely, $G$ and $H$ are homotopy equivalent to $\Omega B G$ and $\Omega B H$ for path connected spaces $B G$ and $B H$, and $G / H$ is the homotopy fiber of a based map $f: B H \longrightarrow B G$. We always assume this much, and we add further hypotheses as needed. Such a framework of generalized homogeneous spaces was first introduced by Rector [10], and a more recent framework of $p$-compact groups has been introduced and studied extensively by Dwyer and Wilkerson [4] and others.

We ask the following question: How similar is the calculation of $H^{*}(G / H ; R)$ to the calculation of the cohomology of classical homogeneous spaces of compact Lie groups? When $R=\mathbf{F}_{p}$ and $H$ is of maximal rank in $G$, in the sense that $H^{*}(H ; \mathbf{Q})$ and $H^{*}(G ; \mathbf{Q})$ are exterior algebras on the same number of generators, the second author has studied the question in 8,9 . There, the fact that $H^{*}(B G ; R)$ need not be a polynomial algebra is confronted and results similar to the classical theorems of Borel and Bott [2, 3] are nevertheless proven. The purpose of this note is to begin to answer the general question without the maximal rank hypothesis, but under the hypothesis that $H^{*}(B G ; R)$ and $H^{*}(B H ; R)$ are polynomial algebras.

In fact, we shall not do any new mathematics. Rather, we shall merely point out that work of the first author [7] that was done before the general context was introduced goes far towards answering the question. Essentially the following theorem was announced in [7] and proven in [5]. We give a brief sketch of its proof and then return to a discussion of its applicability to the question on hand. Let $B T^{n}$ be a classifying space of an $n$-torus $T^{n}$.

Theorem 1. Assume the following hypotheses.

(i) $\pi_{1}(B G)$ acts trivially on $H^{*}(G / H ; R)$.

Received by the editors May 19, 2000.

2000 Mathematics Subject Classification. Primary 55T20, 57T15, 57T35; Secondary 55P35, $55 \mathrm{P} 45$.

The first author was partially supported by the NSF.

(C)2001 American Mathematical Society 
(ii) $R$ is a PID and $H_{*}(B G ; R)$ is of finite type over $R$.

(iii) $H^{*}(B G ; R)$ is a polynomial algebra.

(iv) There is a map e $: B T^{n} \longrightarrow B H$ such that $H^{*}\left(B T^{n} ; R\right)$ is a free $H^{*}(B H ; R)$ module via $e^{*}$.

Then $H^{*}(G / H ; R)$ is isomorphic as an $R$-module to $\operatorname{Tor}_{H^{*}(B G ; R)}\left(R, H^{*}(B H ; R)\right)$, regraded by total degree. Moreover, there is a filtration on $H^{*}(G / H ; R)$ such that its associated bigraded $R$-algebra is isomorphic to $\operatorname{Tor}_{H^{*}(B G ; R)}\left(R, H^{*}(B H ; R)\right)$.

Proof. The first two hypotheses ensure that $H^{*}(G / H ; R)$ is isomorphic to the differential torsion product $\operatorname{Tor}_{C^{*}(B G ; R)}\left(R, C^{*}(B H ; R)\right)$. (See, for example, [5] pp. 2125]. The second hypothesis allows Lemma 3.2 there to be applied with $\mathbf{Z}$ replaced by $R$, thus allowing the finite type over $\mathbf{Z}$ hypothesis assumed there to be replaced by the finite type over $R$ hypothesis assumed here.) Therefore there is an EilenbergMoore spectral sequence that converges from $\operatorname{Tor}_{H^{*}(B G ; R)}\left(R, H^{*}(B H ; R)\right)$ to $H^{*}(G / H ; R)$. The conclusion of the theorem is that this spectral sequence collapses at $E_{2}$ with trivial additive extensions, but not necessarily trivial multiplicative extensions. The last hypothesis and a comparison of spectral sequences argument essentially due to Baum 1] shows that the conclusion holds in general if it holds when $B H=B T^{n}$. (See [5, pp. 37-38].) Here the strange result [5, 4.1] gives that there is a morphism

$$
g: C^{*}\left(B T^{n} ; R\right) \longrightarrow H^{*}\left(B T^{n} ; R\right)
$$

of differential algebras such that $g$ induces the identity map on cohomology and annihilates all $\cup_{1}$-products.

Now the general theory of differential torsion products of 5] kicks in. In modern language, implicit in the discussion of 6, p. 70], there is a model category structure on the category of $A$-modules for any $D G A A$ over $R$ such that every right $A$-module $M$ admits a cofibrant approximation of a very precise sort. Namely, for any $H A$-free resolution $X \otimes_{R} H A \longrightarrow H M$ of $H M$, there is a cofibrant approximation $P=X \otimes_{R} A \longrightarrow M$. Grading is made precise in the cited sources. The essential point is that $P$ is not a bicomplex but rather has differential with many components. When $H A$ is a polynomial algebra and $M=R$, we can take $X$ to be an exterior algebra with one generator for each polynomial generator of $H A$. Here, asssuming that $A$ has a $\cup_{1}$-product that satisfies the Hirsch formula $\left(\cup_{1}\right.$ is a graded derivation), 5, 2.2] specifies the required differential explicitly in terms of $\cup_{1^{-}}$products. Using $g$ to replace $C^{*}\left(B T^{n} ; R\right)$ by $H^{*}\left(B T^{n} ; R\right)$ in our differential torsion product, we see that the differential torsion product $\operatorname{Tor}_{C^{*}(B G ; R)}\left(R, H^{*}\left(B T^{n} ; R\right)\right)$ is computed by exactly the same chain complex as the ordinary torsion product $\operatorname{Tor}_{H^{*}(B G ; R)}\left(R, H^{*}\left(B T^{n} ; R\right)\right)$. (See [5, 2.3].) The conclusion follows.

Hypotheses (i) and (ii) in Theorem 1 are reasonable and not very restrictive. Hypothesis (iii) is intrinsic to the method at hand. Note that $H^{*}(B G ; R)$ can have infinitely many polynomial generators, so that $G$ need not be finite. The key hypothesis is (iv). Here the following homotopical version of a theorem of Borel is relevant. It was first noticed by Rector [10, 2.2] that Baum's proof [1] of Borel's theorem is purely homotopical. A generalized variant of Baum's proof is given in [5, pp. 40-42]. That proof applies directly to give the following theorem. We state it for $H$ and $G$ as in the first paragraph. However, we are interested in its applicability to $T^{n}$ and $H$ in Theorem 1 , and we restate it as a corollary in that special case. 
Theorem 2. Let $R$ be a field and assume the following hypotheses.

(i) $\pi_{1}(B G)$ acts trivially on $H^{*}(G / H ; R)$.

(ii) $H^{*}(B H ; R)$ and $H^{*}(B G ; R)$ are polynomial algebras on the same finite number of generators.

(iii) $H^{*}(G / H ; R)$ is a finite dimensional $R$-module.

Then $H^{*}(G / H ; R) \cong R \otimes_{H^{*}(B G ; R)} H^{*}(B H ; R)$ as an algebra and

$$
H^{*}(B H ; R) \cong H^{*}(B G ; R) \otimes_{R} H^{*}(G / H ; R)
$$

as a left $H^{*}(B G ; R)$-module. In particular, $H^{*}(B H ; R)$ is $H^{*}(B G ; R)$-free.

Corollary 3. Let $R$ be a field and assume given a map e $: B T^{n} \longrightarrow B H$ that satisfies the following properties, where $H / T^{n}$ is the fiber of $e$.

(i) $\pi_{1}(B H)$ acts trivially on $H^{*}\left(H / T^{n} ; R\right)$.

(ii) $H^{*}(B H ; R)$ is a polynomial algebra on $n$ generators.

(iii) $H^{*}\left(H / T^{n} ; R\right)$ is a finite dimensional $R$-module.

Then $H^{*}\left(H / T^{n} ; R\right) \cong R \otimes_{H^{*}(B H ; R)} H^{*}\left(B T^{n} ; R\right)$ as an algebra and

$$
H^{*}\left(B T^{n} ; R\right) \cong H^{*}(B H ; R) \otimes_{R} H^{*}\left(H / T^{n} ; R\right)
$$

as a left $H^{*}(B H ; R)$-module. In particular, $H^{*}\left(B T^{n} ; R\right)$ is $H^{*}(B H ; R)$-free.

When Corollary 3 applies, its conclusion gives hypothesis (iv) of Theorem 1. We comment briefly on applications to the integral and $p$-compact settings for the study of generalized homogeneous spaces.

Remark 4. A counterexample of Rector [10] shows that not all finite loop spaces $H$ have (integral) maximal tori. When $H$ does have a maximal torus, hypothesis (iii) of the corollary holds by definition. Assuming that $H$ is simply connected, $9,3.11]$ describes for which primes $p H^{*}(B H ; \mathbf{Z})$ is $p$-torsion free, so that $H^{*}\left(B H ; \mathbf{F}_{p}\right)$ is a polynomial algebra. If $R$ is the localization of $\mathbf{Z}$ at the primes $p$ for which $H^{*}(H ; \mathbf{Z})$ is $p$-torsion free, then $H^{*}(B H ; R)$ is also a polynomial algebra, and $H^{*}(B T ; R)$ is a free $H^{*}(B H ; R)$-module. That is, hypothesis (iv) of Theorem 1 holds for the localization of $\mathbf{Z}$ away from the finitely many "bad primes" for which $H^{*}\left(B H ; \mathbf{F}_{p}\right)$ is not a polynomial algebra on $n$ generators.

Remark 5. In the $p$-compact setting, taking $R=\mathbf{F}_{p}$, Dwyer and Wilkerson 44,

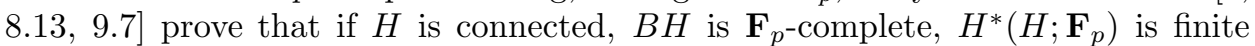
dimensional, and $H^{*}\left(H ; \mathbf{Z}_{p}\right) \otimes \mathbf{z}_{p} \mathbf{Q}$ is an exterior algebra on $n$ generators, then there is a map $e: B T^{n} \longrightarrow B H$ such that $H^{*}\left(H / T^{n} ; \mathbf{F}_{p}\right)$ is finite dimensional. Here Corollary 3 applies whenever $H^{*}\left(B H ; \mathbf{F}_{p}\right)$ is a polynomial algebra on $n$ generators.

\section{REFERENCES}

[1] P.F. Baum. On the cohomology of homogeneous spaces. Topology 7(1968), 15-38. MR 36:2168

[2] A. Borel. Sur la cohomologie des éspaces fibré principaux et des éspaces homogènes de groupes de Lie compact. Ann Math. 57(1953), 115-207. MR 14:490e

[3] R. Bott. An application of Morse theory to the topology of Lie groups. Bull. Soc. Math. France 84(1956), 251-281. MR 19:291a

[4] W.G. Dwyer and C.W. Wilkerson. Homotopy fixed point methods for Lie groups and finite loop spaces. Ann. Math. 139(1994), 395-442. MR 95e:55019

[5] V.K.A.M. Gugenheim and J.P. May. On the theory and applications of differential torsion products. Memoirs Amer. Math. Soc. No. 142, 1974. MR 52:15519

[6] I. Kriz and J.P. May. Operads, algebras, modules, and motives. Astérisque. No. 233. 1995. MR 96j:18006 
[7] J.P. May. The cohomology of principal bundles, homogeneous spaces, and two-stage Postnikov systems. Bull. Amer. Math. Soc. 74(1968), 334-339. MR 39:953

[8] F. Neumann. On the cohomology of homogeneous spaces of finite loop spaces and the Eilenberg-Moore spectral sequence. J. Pure and Applied Algebra 140(1999), 261-287. MR 2000k:55015

[9] F. Neumann. Torsion in the cohomology of finite loop spaces and the Eilenberg-Moore spectral sequence. Topology and its Applications 100(2000), 133-150. MR 2000j:57079

[10] D. Rector. Subgroups of finite dimensional topological groups. J. Pure Appl. Algebra 1(1971), 253-273. MR 46:889

Department of Mathematics, The University of Chicago, Chicago, Illinois 60637

E-mail address: may@math.uchicago.edu

Mathematisches Institut der Georg-August-Universität, Göttingen, Germany

E-mail address: neumann@uni-math.gwdg.de 